\title{
Detection of variable frequency signals using a fast chirp transform
}

\author{
F. A. Jenet and T. A. Prince \\ Division of Physics, Mathematics, and Astronomy and the LIGO Laboratory, California Institute of Technology, \\ Pasadena, California 91125
}

(Received 1 February 2000; published 14 November 2000)

\begin{abstract}
The detection of signals with varying frequency is important in many areas of physics and astrophysics. The current work was motivated by a desire to detect gravitational waves from the binary inspiral of neutron stars and black holes, a topic of significant interest for the new generation of interferometric gravitational wave detectors such as LIGO. However, this work has significant generality beyond gravitational wave signal detection. We define a fast chirp transform (FCT) analogous to the fast Fourier transform. Use of the FCT provides a simple and powerful formalism for detection of signals with variable frequency just as Fourier transform techniques provide a formalism for the detection of signals of constant frequency. In particular, use of the FCT can alleviate the requirement of generating complicated families of filter functions typically required in the conventional matched filtering process. We briefly discuss the application of the FCT to several signal detection problems of current interest.
\end{abstract}

PACS number(s): $04.80 . \mathrm{Nn}, 02.30 . \mathrm{Nw}, 84.40 . \mathrm{Ua}, 95.75 . \mathrm{Pq}$

\section{INTRODUCTION}

The detection of periodic signals is a well-developed art. In contrast, the detection of signals with variable frequency is an active area of research in signal processing. Considerable progress has been made in recent years using a variety of time-frequency techniques which include wavelets, bilinear transforms, and short time Fourier transforms (STFT's) $[1,2]$.

In this paper, we consider the detection of deterministic signals with unknown parameters. The case of deterministic signals with unknown amplitude, phase, frequency, and arrival time has been treated in the literature $[3,4]$. In this paper, we generalize to an arbitrary number of parameters and consider signals with a deterministic, but parametrized, frequency evolution. We call these "variable frequency signals.' Specifically, we consider signals of the form

$$
h_{s}(t)= \begin{cases}A(t) \cos \left(\phi\left(t, \lambda_{0}, \ldots, \lambda_{M}\right)\right), & 0<t<T \\ 0 & \text { otherwise }\end{cases}
$$

where $\phi\left(t, \lambda_{0}, \ldots, \lambda_{M}\right)$ is real and the $\left\{\lambda_{0}, \ldots, \lambda_{M}\right\}$ represent various parameters which describe the phase evolution (e.g. frequency, frequency derivative, etc.). In general, $\phi()$ may depend non-linearly on time. For this paper, the "instantaneous frequency" of $h_{s}$ must be a well-defined quantity and the frequency evolution of the signal must be well resolved in the data. Variable frequency signals include the class of signals usually known as chirps, i.e. signals which have a monotonically increasing or decreasing instantaneous frequency. Such signals appear in many contexts, such as almost-periodic signals with a small frequency drift or periodic signals emitted from accelerated systems. Chirps are discussed often in the literature of signal processing $[5,4,2]$. We will concentrate on chirp signals in order to simplify the description of the chirp transform algorithm.

A standard technique for the detection of signals in the presence of noise is the "matched filter" technique $[3,6]$.
Detection of a signal $h_{s}(t)$ in the presence of white noise in a data stream $h(t)$ of length $T$ is based on the matched filter output:

$$
\mathcal{S}=\int_{0}^{T} d t h_{s}(t) h(t)=\int_{0}^{T} d \tau h_{f i l t}(\tau) h(T-\tau)
$$

where $h_{f i l t}(t)$ is an optimal filter function in the time domain. For white noise, the optimum filter has an impulse response given by $h_{f i l t}(\tau)=h_{s}(T-\tau)$, in the interval $0<\tau$ $<T$. To detect a signal beginning at time $t_{0}$ in a data stream of arbitrary length we compute the quantity:

$$
\mathcal{S}\left(t_{0}\right)=\int_{-\infty}^{\infty} d \tau h_{f i l t}(\tau) h\left[\left(t_{0}+T\right)-\tau\right]
$$

where $h(t)$ is zero outside a finite region of interest. When colored noise is present, it is conventional to work in the frequency domain. For matched filtering of real signals with an unknown arrival time, one constructs a signal estimator of the form

$$
\mathcal{S}\left(t_{0}\right)=4 \operatorname{Re}\left[\int_{0}^{\infty} d f \frac{\tilde{h}(f) \tilde{h}_{s}^{*}(f) e^{-i 2 \pi f t_{0}}}{S_{h}(f)}\right],
$$

where $\widetilde{h}(f)$ is the Fourier transform of the signal plus noise, $h(t)$, defined as

$$
\tilde{h}(f)=\int_{-\infty}^{\infty} d t h(t) e^{i 2 \pi f t}
$$

$\tilde{h}_{s}(f)$ is the Fourier transform of the signal waveform and $S_{h}(f)$ is the one-sided noise power spectral density. An optimum filter output is calculated for each realization of $h_{s}$ from Eq. (1) using different values of the parameters. The approach of matched filtering thus requires the construction of a "dense" set of signal waveforms which cover the parameter space of possible signals. 
Other approaches to the variable frequency detection problem include techniques based on either synthesizing a multichannel filterbank [7] or resampling the data at a variable rate $[8,9]$. These techniques are used widely in the radio pulsar community and, like the conventional matched filtering approach described above, differ significantly from the algorithm presented here.

Just as the Fourier transform can be considered a form of matched filtering using a dense set of sine and cosine functions appropriate for periodic signals of unknown amplitude and phase, we wish to define a transform which performs matched filtering for a dense set of variable frequency signals with unknown parameters. By using the Fourier transform for periodic signals, we avoid the need of explicitly storing and computing the individual filter functions, i.e. the sine and cosine functions in the time domain. Analogously, the appropriate transform for variable frequency signals will avoid the need of generating a large set of filter functions and will provide a prescription for densely covering the set of possible signal waveforms. We will informally call the transform for variable frequency waveforms a "chirp transform."

The term "chirp transform" has been used elsewhere in the literature. For instance, Oppenheim et al. [10] describe a "chirp transform algorithm" which is a special case of the "chirp-z transform." The chirp-z transform is well-known and can be used to evaluate quadratic chirps. The method described in our paper is general and not constrained to quadratic chirp functions. We call the algorithm described in this paper the "fast chirp transform" (FCT).

The techniques discussed in this paper appear to be related to filter bank design, to wavelet analysis, and to STFT's. In fact, the chirp transform can be viewed as a prescription for coherently adding the outputs of a bank of variable-length STFT's with a particular time-domain relationship. Thus, the fast chirp transform may already exist in some other formalism in the signal processing literature, but we are currently unaware of it. The work of Schutz [11] and of Williams and Schutz [12] describes an approach which is similar in several aspects, but differs in that constant length STFT's are used rather than variable-length transforms.

In Sec. II, we give the discrete forms of the matched filter outputs and discuss how these may be expressed in the form of the discrete analog to generalized Fourier integrals. In Sec. III we derive the two-parameter fast chirp transform that can be used to evaluate the discrete matched filter expressions. In Sec. IV we generalize the definition of the FCT to an arbitrary number of parameters. In Sec. V we briefly discuss several applications of the fast chirp transform.

\section{DISCRETE MATCHED FILTERING}

The discrete forms of the time-domain and frequency domain matched filter outputs [Eqs. (2) and (4)] are given by

$$
\begin{aligned}
& \mathcal{S}\left(\lambda_{0}, \ldots, \lambda_{M}\right) \\
& \quad=\sum_{j=0}^{N_{0}-1} h_{s}\left(j, \lambda_{0}, \ldots, \lambda_{M}\right) h(j), \quad \text { and }
\end{aligned}
$$

$$
\begin{aligned}
& \mathcal{S}\left(j_{0}, \lambda_{0}, \ldots, \lambda_{M}\right) \\
& \quad=\frac{4}{N_{0}} \operatorname{Re}\left[\sum_{k_{0}=0}^{N_{0}-1} \frac{\tilde{h}\left(k_{0}\right) \tilde{h}_{s}^{*}\left(k_{0}, \lambda_{0}, \ldots, \lambda_{M}\right) e^{-i 2 \pi j_{0} k_{0} / N_{0}}}{S_{h}\left(k_{0}\right)}\right] .
\end{aligned}
$$

The Appendix shows that for variable frequency waveforms, $h_{s}(t)=A(t) \cos \left(\phi\left(t, \lambda_{0}, \ldots, \lambda_{M}\right)\right)$, the discrete matched filter outputs can be expressed as generalized Fourier integrals, and in discrete notation take on a particularly simple form:

$$
\begin{gathered}
\mathcal{S}\left(\lambda_{0}, \ldots, \lambda_{M}\right)=\operatorname{Re}\left[\sum_{j_{0}=0}^{N_{0}-1} \mathcal{G}_{j_{0}} e^{-i \phi\left(j_{0}, \lambda_{0}, \ldots, \lambda_{M}\right)}\right], \\
\mathcal{S}\left(j_{0}, \lambda_{0}, \ldots, \lambda_{M}\right)=\frac{4}{N_{0}} \operatorname{Re}\left[\sum_{k_{0}=0}^{N_{0}-1} \tilde{\mathcal{H}}_{k_{0}} e^{-i \Phi\left(k_{0}, j_{0}, \lambda_{0}, \ldots, \lambda_{M}\right)}\right],
\end{gathered}
$$

where $\mathcal{G}_{j_{0}}$ and $\widetilde{\mathcal{H}}_{k_{0}}$ can be considered as the time or frequency series to be transformed, and $\Phi\left(k_{0}, j_{0}, \lambda_{0}, \ldots, \lambda_{M}\right)$ is a real phase function of the form given in Eq. (A9).

In the next section we define the chirp transform and show how it can be used to evaluate discrete transforms of the type shown in Eqs. (8) and (9). Appropriate forms of the FCT will replace both the forward and inverse transforms contained in Eqs. (8) and (9). An inverse FCT is not applicable to the detection problem and will not be considered in this paper. The above formulation of the matched filtering process included the starting phase as a search parameter. An alternative approach is to convolve the signal with both the in-phase, $\cos (\phi)$, and quadrature-phase, $\sin (\phi)$, filters and then sum the squares of the results. This formulation is independent of the starting phase. In order to make the chirp transform applicable to both formulations and to a wider class of problems in general, the $\mathrm{Re}[$ ] operator will not be included in the definition. If necessary, this operator can be applied after the transform.

\section{THE TWO-PARAMETER FAST CHIRP TRANSFORM}

\section{A. Example: quadratic chirp (linear frequency drift)}

As an initial example, we consider the problem of the detection of a quadratic chirp, e.g. a signal of the form $h_{s}(t)=A(t) \cos \left(2 \pi\left(f t+\frac{1}{2} \dot{f} t^{2}\right)\right)$. We wish to detect this signal by matched filtering with a dense set of quadratic chirp waveforms. This requires evaluation of the sums of the form in Eq. (6). Note that if $\dot{f}$ were zero, the signal waveform would be periodic and we might discretely sample the input signal and then compute a power spectral estimate using the fast Fourier transform (FFT).

Here we define a FCT for the quadratic chirp analogous to the FFT. This definition will be generalized to an arbitrary parameter frequency waveform in the next section. For simplicity we first define the discrete chirp transform (DCT) for the quadratic chirp in analogy with the discrete Fourier transform (DFT): 


$$
H_{\left\{k_{0}, k_{1}\right\}}=\sum_{j_{0}=0}^{N_{0}-1} h_{j_{0}} e^{i 2 \pi\left[k_{0}\left(j_{0} / N_{0}\right)+k_{1}\left(j_{0} / N_{0}\right)^{2}\right]},
$$

where $h_{j_{0}}$ is the discretely sampled data. The quadratic nature of the chirp is specified by the term $\left(j_{0} / N_{0}\right)^{2}$ in the exponential. If $k_{1}$ is zero, we have the usual DFT.

To derive the fast chirp transform, we begin by breaking the interval $\left\{j_{0}=0, \ldots, N_{0}-1\right\}$ into a set of contiguous subintervals

$$
H_{\left\{k_{0}, k_{1}\right\}}=\sum_{j_{1}=0}^{N_{1}-1} \sum_{j_{0}=j_{0}^{\min }\left(j_{1}\right)}^{j_{0}^{\min }\left(j_{1}+1\right)-1} h_{j_{0}} e^{i 2 \pi\left[k_{0}\left(j_{0} / N_{0}\right)+k_{1}\left(j_{0} / N_{0}\right)^{2}\right]},
$$

where $j_{0}^{\min }\left(j_{1}\right)$ is the lower boundary of each of the intervals. The requirement that the intervals be contiguous implies $j_{0}^{\text {min }}\left(j_{1}+1\right)=j_{0}^{\text {min }}\left(j_{1}\right)+n_{0}\left(j_{1}\right)$, where $n_{0}\left(j_{1}\right)$ is the number of points in the interval.

The boundaries are chosen as follows: we demand that the term $k_{1}\left(j_{0} / N_{0}\right)^{2}$ change by no more than $\pi$ for values of $k_{1}$ appropriate for the quadratic chirp signals being considered. If the term $k_{1}\left(j_{0} / N_{0}\right)^{2}$ changes by more than $\pi$ over a single sample, then the signal is not considered to be finely enough sampled to resolve the phase evolution. This limitation is analogous to the Nyquist sampling limit for periodic signals. Requiring that the term $k_{1}\left(j_{0} / N_{0}\right)^{2}$ remain relatively constant over a sub-interval allows us to approximate the DCT as

$$
\begin{aligned}
H_{\left\{k_{0}, k_{1}\right\}} \approx & \sum_{j_{1}=0}^{N_{1}-1} e^{i 2 \pi\left[k_{1}\left(j_{0}^{\min }\left(j_{1}\right) / N_{0}\right)^{2}\right]} \\
& \times \sum_{j_{0}=j_{0}^{\text {min }}\left(j_{1}\right)}^{j_{0}^{\text {min }}\left(j_{1}+1\right)-1} h_{j_{0}} e^{i 2 \pi k_{0}\left(j_{0} / N_{0}\right)}
\end{aligned}
$$

and we note that the second summation can now be computed as a fast Fourier transform. We further demand that the term $k_{1}\left(j_{0}^{\min }\left(j_{1}\right) / N_{0}\right)^{2}$ increment by a constant amount from one sub-interval to the next. The requirement that the increment be less than $\pi$ for the maximum value of $k_{1}, k_{1}^{\max }$, specifies $N_{1}=2 k_{1}^{\max }$, with the "Nyquist" restriction that $k_{1}^{\max } \leqslant N_{0} / 4$ for the case of a quadratic chirp. We can then write

$$
H_{\left\{k_{0}, k_{1}\right\}} \approx \sum_{j_{1}=0}^{N_{1}-1} e^{i 2 \pi k_{1}\left(j_{1} / N_{1}\right)} \sum_{j_{0}=j_{0}^{\text {min }}\left(j_{1}\right)}^{j_{0}^{\text {min }}\left(j_{1}+1\right)-1} h_{j_{0}} e^{i 2 \pi k_{0}\left(j_{0} / N_{0}\right)}
$$

where we have anticipated that we will evaluate the sum for integral values of $k_{1}$ using a standard FFT. Finally, we express the inner sum in standard FFT form by extracting a phase factor and we define the two-parameter FCT, $C_{\left\{k_{0}, k_{1}\right\}}$, as

$$
\begin{aligned}
H_{\left\{k_{0}, k_{1}\right\}} \approx & C_{\left\{k_{0}, k_{1}\right\}} \\
\equiv & \sum_{j_{1}=0}^{N_{1}-1} e^{i 2 \pi k_{1}\left(j_{1} / N_{1}\right)}\left[e^{i 2 \pi k_{0}\left[j_{0}^{m i n}\left(j_{1}\right) / N_{0}\right]}\right. \\
& \left.\times \sum_{j_{0}=0}^{N_{0}\left(j_{1}\right)-1} h_{j_{0}+j_{0}^{m i n}\left(j_{1}\right)} e^{i 2 \pi k_{0}\left(j_{0} / N_{0}\right)}\right] .
\end{aligned}
$$

As before, $n_{0}\left(j_{1}\right)$ is the number of points in the interval. We call $k_{0}$ and $k_{1}$ the "conjugate variables" of the linear and quadratic terms, respectively, in the same sense that frequency and time are a conjugate variable pair. Note that both summations can be implemented using FFT's and thus we are justified in calling the transform a fast chirp transform. The non-linearity of the quadratic chirp is absorbed into the specification of the boundaries of the contiguous intervals. This is the key concept of the FCT. Note that the definition of $C_{\left\{k_{0}, k_{1}\right\}}$ is general and does not depend explicitly on the quadratic nature of the phase evolution. The same definition will apply to other non-linear phase evolution functions.

We may also write

$$
C_{\left\{k_{0}, k_{1}\right\}}=\sum_{j_{1}=0}^{N_{1}-1} e^{i 2 \pi k_{1}\left(j_{1} / N_{1}\right)} C_{\left\{k_{0}, j_{1}\right\}}
$$

where we have used the notation $C_{\left\{k_{0}, j_{1}\right\}}$ to indicate that it is a partial transform, i.e. transformed over one index, $j_{0}$, but not the other, $j_{1}$. Equation (15) illustrates an interesting feature of the FCT, which is important in implementation considerations. Although the number of points in an interval, $n_{0}\left(j_{1}\right)$, may be small, the partial transform, $C_{\left\{k_{0}, j_{1}\right\}}$, requires evaluation at a large number of values of $k_{0}$, for example at $N_{0}$ values of $k_{0}$. This is equivalent to calculating the oversampled FFT of the individual intervals with an oversampling factor of $N_{0} / n_{0}\left(j_{1}\right)$. The problem of computing the $C_{\left\{k_{0}, j_{1}\right\}}$ thus reduces to the problem of estimating the oversampled spectrum of each interval. The oversampled spectrum may be calculated exactly using the fractional Fourier transform (FRFT). Fast methods have been developed for evaluating FRFT's [13] and it can be shown that the calculation of the values of the individual oversampled FFT's that enter into $C_{\left\{k_{0}, j_{1}\right\}}$ in Eqs. (14) and (15) requires $\mathrm{O}\left(N_{0} \log _{2} n_{0}\left(j_{1}\right)\right)$ operations to be computed exactly. The computation required to calculate the entire set of FRFT's can be shown to be $\mathrm{O}\left(N_{1} N_{0} \log _{2}\left(N_{0} / N_{1}\right)\right)$. The phase factors, $\exp \left[i 2 \pi k_{0}\left(j_{0}^{\min }\left(j_{1}\right) / N_{0}\right)\right]$ in Eq. (14) are easily computed as part of the same formalism.

Taking into account the evaluation of the inner and outer sums separately, the number of operations required for the evaluation of the FCT can be shown to be

$$
N_{o p s} \leqslant \mathrm{O}\left(N_{1} N_{0} \log _{2} N_{0}\right)
$$

which is at least as efficient as the matched filter approach. The inequality indicates that the evaluation requires less computations if approximations are used in evaluating the oversampled FFT's or if a coarser sampling of the FCT over 
$k_{0}$ values is used. We will discuss the issue of computational efficiency further in Sec. III D below.

\section{B. Accuracy of the approximations}

How accurately can the FCT approximate the discrete matched filter output? There are three types of approximations to be considered: (1) Possible use of the stationary phase approximation in deriving a form for the matched filter output; (2) The constant phase approximation used to derive the discrete form of the FCT; and (3) Possible use of approximations in calculating the oversampled FFT's that enter into the FCT. We will consider each of these approximations in turn.

(1) There may be some error due to the stationary phase approximation itself (Appendix) if this is used to approximate the Fourier transform of the signal waveform. This is not inherently part of the FCT, and we will not discuss this approximation here since it depends on the specific waveform being analyzed. However, we note that the stationary phase approximation can be extremely accurate in practice $[14,15]$.

(2) The error due to the constant phase approximation [Eq. (12)] in the FCT itself is obviously dependent on the value of the conjugate variables at which the FCT is evaluated. If the value of $k_{1}$ is small, the value of the increase in the quadratic phase term as $j_{1}$ goes to $j_{1}+1$ is also small, and the approximation will be very accurate. By analogy to the Fourier transform, we expect the "frequency response" of the output of the matched filter computed by the FCT to behave similarly to the frequency response of a power spectrum computed with an FFT. Specifically, just as there is a roll-off in power for some periodic signals near the Nyquist frequency, there will be a roll-off in the accuracy of the FCT approximation to the matched filter output for signals near the Nyquist limit of the conjugate variable, namely $k_{1}$ (Nyquist) $=N_{1} / 2$. For values of $k_{1}$ well below Nyquist, we expect the accuracy of the FCT approximation to the matched filter output to be very good. Furthermore, if we wish higher accuracy in the FCT approximation, we can employ the same techniques used in Fourier analysis, namely, we can "oversample" the FCT. This can be accomplished by "zero-padding" of the outer $\left(j_{1}\right)$ FFT.

(3) Finally, there may be errors due to the possible use of approximations in calculating the oversampled FFT's. As indicated in Eq. (16), the exact calculation of the oversampled FFT's requires $\mathrm{O}\left(N_{1} N_{0} \log _{2} N_{0}\right)$ calculations. However, this assumes that each interval is oversampled by a factor $N_{0} / N_{0}\left(j_{1}\right)$, which can be quite large. Typically, oversampling factors of $2^{2}-2^{3}$ are sufficient for an accurate approximation. If $N_{1}$ is large, the computational requirements may thus be reduced considerably by using such approximations [i.e. $\mathrm{O}\left(N_{0} \log _{2} N_{0}\right)$ rather than $\mathrm{O}\left(N_{1} N_{0} \log _{2} N_{0}\right)$ ]. A quantitative discussion of oversampling approximations is beyond the scope of this paper.

We also remark that the FCT formalism lends itself naturally to a variety of hierarchical search approaches. For instance, consider Eqs. (14) and (15). The outer sum is a coherent addition of the contributions from the individual intervals. In order to implement a fast hierarchical search, we could simply take the magnitudes of each $C_{\left\{k_{0}, j_{1}\right\}}$ and add them as an incoherent sum over $j_{1}$, giving a measure of the incoherent power as a function of $k_{0}$. Values of $k_{0}$ with significant incoherent power could then be examined in more detail by performing the coherent summation over $j_{1}$. This could lead to a dramatic decrease in the required number of computations, depending on the threshold set for the incoherent power summation step.

\section{Implementation considerations}

As an initial trial, we have implemented the twoparameter FCT and tested it on several types of waveforms, including the quadratic chirp discussed above and the "Newtonian chirp" discussed in Sec.V A below. We have used two implementations, one which uses a fixed length oversampling of the inner FFT, and one which uses a prepackaged 2D FFT algorithm.

In this section we will describe in more detail the 2D FFT implementation in order to give further insight into the details of the two-parameter FCT algorithm. The 2D FFT implementation is extremely simple to code although it is not the most computationally efficient. It will be shown in the next section that even so, it is nearly as efficient as the bruteforce matched filtering method.

The 2D FFT implementation of the FCT packs the initial one dimensional data array, $h_{j_{0}}$, into a sparse two dimensional array $\hat{h}_{j_{0}, j_{1}}$. The packing will be determined by the $j_{0}^{\min }\left(j_{1}\right)$ array which is ultimately defined by the phase function $\phi\left(j_{0}\right)$. Once the data are packed appropriately, the FCT is calculated using any pre-packaged 2D FFT routine. For the rest of this discussion, it is assumed that $N_{0}$ and $N_{1}$ are compatible with the 2D FFT routine being employed. This usually means that these lengths are a power of two.

From Eq. (13), the two parameter FCT may be written as

$$
C_{\left\{k_{0}, k_{1}\right\}}=\sum_{j_{1}=0}^{N_{1}-1} e^{i 2 \pi k_{1}\left(j_{1} / N_{1}\right)} \sum_{j_{0}=j_{0}^{j_{0} \text { min }}\left(j_{1}\right)}^{\left.j_{1}+1\right)-1} h_{j_{0}} e^{i 2 \pi k_{0}\left(j_{0} / N_{0}\right)} .
$$

By defining a two dimensional $N_{0} \times N_{1}$ array such that

$$
\hat{h}_{j_{0}, j_{1}}= \begin{cases}h_{j_{0}}, & j_{0}^{\min }\left(j_{1}\right) \leqslant j_{0}<j_{0}^{\min }\left(j_{1}+1\right), \\ 0 & \text { otherwise }\end{cases}
$$

Eq. (17) becomes

$$
C_{\left\{k_{0}, k_{1}\right\}}=\sum_{j_{1}=0}^{N_{1}-1} \sum_{j_{0}=0}^{N_{0}-1} \hat{h}_{j_{0}, j_{1}} e^{i 2 \pi\left(k_{1} j_{1} / N_{1}+k_{0} j_{0} / N_{0}\right)}
$$

As promised, $C_{\left\{k_{0}, k_{1}\right\}}$ is the 2D FFT of the sparsely packed array $\hat{h}_{j_{0}, j_{1}}$. For the case of a monotonic phase function that satisfies $\phi(0)=0$ and $\phi\left(N_{0}\right)=N_{1}$, the array boundaries are given by

$$
j_{0}^{\min }\left(j_{1}\right)=\phi^{-1}\left(j_{1}\right)
$$


Note that we have the freedom to rescale $\phi(j)$ so that $N_{1}$ may be chosen arbitrarily.

The number of operations needed by the FCT implementation using a 2D FFT is the same as that needed by a two dimensional FFT of order $N_{0} \times N_{1}$ :

$$
N_{f c t-2 D}=\mathrm{O}\left(N_{0} N_{1} \log _{2} N_{0} N_{1}\right) .
$$

\section{Computational efficiency}

For a matched filter operation using individual filters, there is one Fourier transform to perform for each filter, thus the number of computations, $N_{m f}$, is of order

$$
N_{m f}=\mathrm{O}\left(N_{1} N_{0} \log _{2} N_{0}\right),
$$

where $N_{1}$ is the number of filter functions needed to cover the space of possible waveforms, and $N_{0}$ is the number of samples in the time series or frequency spectrum. As shown in Sec. III A using the fractional Fourier transform (FRFT), the FCT computation can be of order

$$
N_{f c t-F R F T} \leqslant \mathrm{O}\left(N_{1} N_{0} \log _{2} N_{0}\right) .
$$

Two approaches offer potential computational gains: (1) Reducing the oversampling factor in computing the inner FFT (discussed in Sec. III A), and (2) relaxing the requirement of full resolution in the $k_{0}$ variable. Reducing the oversampling factor can change the inner sum computation from an $\mathrm{O}\left(N_{0} N_{1} \log _{2}\left(N_{0} / N_{1}\right)\right)$ calculation to an $\mathrm{O}\left(N_{0} \log _{2} N_{0}\right)$ calculation (see Sec. III A). If $N_{1} \gg 1$ the total calculation of the FCT will then be of order $\mathrm{O}\left(N_{0} N_{1} \log _{2} N_{1}\right)$. This is potentially a factor of $\mathrm{O}\left(\log _{2} N_{0} / \log _{2} N_{1}\right)$ more efficient than the conventional matched filtering technique. We emphasize that the detailed coefficients in front of the scalings are not yet known for computationally efficient implementations.

Significant computational gains are also potentially available by relaxing the requirement of full resolution in the $k_{0}$ variable. Not all problems require the high $k_{0}$ resolution that the previously discussed FCT implementations deliver. While the power is very localized in the $k_{0}$ variable when the value of $k_{1}$ is that of the actual signal (and vice-versa), the power can be significant for values of $k_{0}$ and $k_{1}$ which simultaneously deviate from the actual signal values. The reason for this is that the deviation in $k_{0}$ can be compensated for by a deviation in $k_{1}$, providing a reasonable correlation of the matched filter template with the actual signal. The determination of the optimum sampling resolution is closely related to the calculation of the ambiguity function [3]. Discussions of techniques for calculating the ambiguity function have been given by Owen [16], Mohanty and Dhurandhar [17], and Owen and Sathyaprakash [18] for special cases of the types of chirp functions considered here.

The FCT may be evaluated at lower resolution by reducing the order of the 2D array $\hat{h}_{j_{0}, j_{1}}$ (Sec. III D) from $N_{0}$ $\times N_{1}$ to $\left(\epsilon N_{0}\right) \times N_{1}$ where $\epsilon<1$. As long as $\left(\epsilon N_{0}\right) \times N_{1}$ $>N_{0}$, we can still pack the original $N_{0}$ data points into this smaller array. The resulting FCT will have a coarser $k_{0}$ resolution but it will take $\mathrm{O}\left(\epsilon N_{1} N_{0} \log _{2} N_{0}\right)$ operations to perform (again neglecting terms of order $\log _{2} N_{1}$ ). Alternatively, in the straightforward evaluation of the FCT contained in Eq. (15), the sums over $j_{1}$ are simply evaluated at a decimated set of $k_{0}$.

We remark that sampling the $\mathrm{FCT}$ at lower resolution can be used as part of a hierarchical algorithm, i.e. the FCT is first calculated at lower resolution and values of $k_{0}$ are identified having excess signal strength. The FCT is then evaluated on a finer grid of $k_{0}$ values near those values of $k_{0}$ exhibiting the excess signal.

\section{E. Selection of the range for evaluation of the FCT}

We can select ranges of $k_{0}$ and $k_{1}$ to be evaluated by a process analogous to a heterodyne operation on a periodic signal. Selection of the range of $k_{0}$ is done by the usual process of down-conversion and low-pass filtering and we will not consider it further here. For the parameter $k_{1}$ the process is different in that no low-pass filtering is required, and because the range of $k_{1}$ can depend on $k_{0}$, i.e. the conjugate parameter ranges need not be independent. This provides considerable flexibility in determining the shape and volume of parameter space that can be efficiently searched.

Suppose we wish to compute the FCT for a range of $k_{1}$ centered on $k_{1}^{\text {mid }}$. Re-writing the two-parameter FCT including the $k_{1}^{\text {mid }}$ term, we obtain

$$
H_{\left\{k_{0}, k_{1}\right\}}=\sum_{j_{0}=0}^{N_{0}-1} h_{j_{0}} e^{i 2 \pi\left[k_{0}\left(j_{0} / N_{0}\right)+\left(k_{1}+k_{1}^{m i d}\left(k_{0}\right)\right)\left(j_{0} / N_{0}\right)^{2}\right]},
$$

where we have explicitly allowed $k_{1}^{\text {mid }}$ to depend on $k_{0}$. As before, assuming that $k_{1}+k_{1}^{\text {mid }}$ is sufficiently small, we take the quadratic phase term out of the inner summation to obtain

$$
\begin{aligned}
H_{\left\{k_{0}, k_{1}\right\}} \approx & C_{\left\{k_{0}, k_{1}\right\}} \\
= & \sum_{j_{1}=0}^{N_{1}-1} e^{i 2 \pi k_{1}\left(j_{1} / N_{1}\right)} \\
& \times\left[e^{i 2 \pi\left[k_{0}\left(j_{0}^{\text {min }}\left(j_{1}\right) / N_{0}\right)+k_{1}^{m i d}\left(k_{0}\right)\left(j_{1} / N_{1}\right)\right]}\right. \\
& \left.\times \sum_{j_{0}=0}^{N_{0}\left(j_{1}\right)-1} h_{j_{0}+j_{0}^{m i n}\left(j_{1}\right)} e^{i 2 \pi k_{0}\left(j_{0} / N_{0}\right)}\right] .
\end{aligned}
$$

To further simplify the notation for the outer sum, we define

$$
\Theta\left(k_{0}, j_{1}, j_{0}^{\text {min }}, k_{1}^{\text {mid }}\right)=k_{0}\left[j_{0}^{\text {min }}\left(j_{1}\right) / N_{0}\right]+k_{1}^{\text {mid }}\left(k_{0}\right)\left(j_{1} / N_{1}\right)
$$

to obtain

$$
\begin{aligned}
C_{\left\{k_{0}, k_{1}\right\}}= & \sum_{j_{1}=0}^{N_{1}-1} e^{i 2 \pi k_{1}\left(j_{1} / N_{1}\right)}\left[e^{i 2 \pi \Theta\left(k_{0}, j_{0}^{m i n}, k_{1}^{m i d}\right)}\right. \\
& \left.\times \sum_{j_{0}=0}^{N_{0}\left(j_{1}\right)-1} h_{j_{0}+j_{0}^{m i n}\left(j_{1}\right)} e^{i 2 \pi k_{0}\left(j_{0} / N_{0}\right)}\right] .
\end{aligned}
$$


It is important to note that $N_{1}$ can be chosen to be any integer less than or equal to the "Nyquist limit," $N_{0} / 2$. This is due to the fact that we can choose the boundaries, $j_{0}^{\min }\left(j_{1}\right)$, of the intervals arbitrarily as long as the phase change from the quadratic term is kept sufficiently small. Thus, we are free to choose any range of $k_{1}$ around $k_{1}^{\text {mid }}$ as long as the size of the range does not exceed the Nyquist limit.

\section{GENERALIZED DEFINITION OF THE FCT}

Because the non-linearities of the phase evolution enter into the FCT only in the boundaries of the sub-intervals, we may generalize the definition of the FCT to the case of a sum of non-linear phase evolution terms. In particular, consider the DCT:

$$
\begin{aligned}
& H_{\left\{k_{0}, \ldots, k_{M-1}\right\}} \\
& \quad=\sum_{j_{0}=0}^{N_{0}-1} h_{j_{0}} \exp \left[i 2 \pi\left(k_{0} \frac{j_{0}}{N_{0}}+\sum_{p=1}^{M-1} k_{p} \frac{\phi_{p}\left(j_{0}\right)}{\phi_{p}\left(N_{0}\right)}\right)\right],
\end{aligned}
$$

where the $\left\{\phi_{p}\left(j_{0}\right): p=1, \ldots, M-1\right\}$ are a set of parameterless, non-linear phase functions. The corresponding FCT is

$$
\begin{aligned}
C_{\left\{k_{0}, \ldots, k_{M-1}\right\}}= & \sum_{j_{M-1}=0}^{N_{M-1}-1} e^{i 2 \pi k_{M-1}\left[\left(j_{M-1}\right) /\left(N_{M-1}\right)\right]} \ldots \exp \left[i 2 \pi k_{1}\left(\frac{j_{1}^{\min }\left(j_{2}, \ldots, j_{M-1}\right)}{N_{1}}\right)\right] \\
& \times \sum_{j_{1}=0}^{N_{1}\left(j_{2}, \ldots, j_{M-1}\right)-1} e^{i 2 \pi k_{1}\left(j_{1} / N_{1}\right)} \exp \left[i 2 \pi k_{0}\left(\frac{j_{0}^{\min }\left(j_{1}, \ldots, j_{M-1}\right)}{N_{0}}\right)\right] \\
& \times \sum_{j_{0}=0}^{N_{0}\left(j_{1}, \ldots, j_{M-1}\right)-1} e^{i 2 \pi k_{0}\left(j_{0} / N_{0}\right)} h_{j_{0}+j_{0}^{\min }\left(j_{1}, \ldots, j_{M-1}\right)}
\end{aligned}
$$

where the $j_{p}^{\min }\left(j_{p+1}, \ldots, j_{M-1}\right)$ and $N_{p}\left(j_{1}, \ldots, j_{M-1}\right)$ are specified by the phase functions $\left\{\phi_{p}\left(j_{0}\right): p=1, \ldots, M-1\right\}$, and the $\left\{N_{p}: p=1, \ldots, M-1\right\}$ are determined by the maximum allowed values of the $\left\{k_{p}: p=1, \ldots, M-1\right\}$. As before, the intervals specified by the $j_{0}^{\min }\left(j_{1}, \ldots, j_{M-1}\right)$ are contiguous. Thus for each non-linear phase function $\phi_{p}\left(j_{0}\right)$ there is a corresponding "conjugate variable," $k_{p}$.

In analogy with Eqs. (18) and (19), the M parameter FCT may be written in the form of an M-dimensional discrete Fourier transform by defining the matrix $\hat{h}_{j_{0}, \ldots, j_{M-1}}$ such that

$$
\hat{h}_{j_{0}, \ldots, j_{M-1}}= \begin{cases}h_{j_{0}} & j_{p} \leqslant \frac{\phi_{p}\left(j_{0}\right) N_{p}}{\phi_{p}\left(N_{0}\right)}<j_{p}+1 \text { for all } p \in[1, M-1] \\ 0 & \text { otherwise. }\end{cases}
$$

With this definition, Eq. (29) becomes

$$
C_{\left\{k_{0}, \ldots, k_{M-1}\right\}}=\sum_{j_{M-1}=0}^{N_{M-1}-1} \ldots \sum_{j_{0}=0}^{N_{0}-1} e^{i 2 \pi\left(\frac{k_{M-1} j_{M-1}}{N_{M-1}}+\cdots+\frac{k_{0} j_{0}}{N_{0}}\right)} \hat{h}_{j_{0}, \ldots, j_{M-1}} .
$$

Note that the interval boundaries, $j_{p}^{\min }$, may be determined from Eq. (30).

Finally, we consider how the range for evaluation of the generalized FCT can be specified, analagous to Eq. (27) for the two-parameter case. To specify the region for which the FCT is to be evaluated, we add a term to each of the exponential terms in Eq. (29). As for the two-parameter case, Eq. (26), we define

$$
\Theta_{p}\left(k_{p-1}, j_{p}, j_{p-1}^{\min }, k_{p}^{\text {mid }}\right)=k_{p-1}\left(j_{p-1}^{\min } / N_{p-1}\right)+k_{p}^{\text {mid }}\left(j_{p} / N_{p}\right)
$$

where $j_{p-1}^{\text {min }}$ is a function of $\left\{j_{p}, \ldots, j_{M-1}\right\}$ and $k_{p}^{\text {mid }}$ can depend on $\left\{k_{0}, k_{1}+k_{1}^{\text {mid }}, \ldots, k_{p-1}+k_{p-1}^{\text {mid }}\right\}$. The parameter space searched by the FCT will then be $k_{p}^{\text {mid }} \pm N_{p} / 2$. This provides considerable flexibility in determining the shape and volume of parameter space that can be efficiently searched. We can now write

$$
C_{\left\{k_{0}, \ldots, k_{M-1}\right\}}=\sum_{j_{M-1}=0}^{N_{M-1}-1} e^{i 2 \pi k_{M-1}\left[\left(j_{M-1}\right) /\left(N_{M-1}\right)\right]} e^{i 2 \pi \Theta_{M-1}\left(k_{M-2}, j_{M-1}, j_{M-2}^{m i n}, k_{M-1}^{m i d}\right)} \sum_{j_{M-2}=0}^{n_{M-2}\left(j_{M-1}\right)-1} e^{i 2 \pi k_{M-2}\left[\left(j_{M-2}\right) /\left(N_{M-2}\right)\right]}
$$




$$
\begin{aligned}
& \times \cdots \\
& \times e^{i 2 \pi \Theta_{2}\left(k_{1}, j_{2}, j_{1}^{m i n}, k_{2}^{\text {mid }}\right)} \sum_{j_{1}=0}^{n_{1}\left(j_{2}, \ldots, j_{M-1}\right)-1} e^{i 2 \pi k_{1}\left(j_{1} / N_{1}\right)} e^{i 2 \pi \Theta_{1}\left(k_{0}, j_{1}, j_{0}^{m i n}, k_{1}^{\text {mid }}\right)} \\
& \times \sum_{j_{0}=0}^{n_{0}\left(j_{1}, \ldots, j_{M-1}\right)-1} e^{i 2 \pi k_{0}\left(j_{0} / N_{0}\right)} h_{j_{0}+j_{0}^{m i n}\left(j_{1}, \ldots, j_{M-1}\right)}
\end{aligned}
$$

\section{DISCUSSION: APPLICATION TO DETECTION OF VARIABLE FREQUENCY SIGNALS}

\section{A. Detection of gravitational waves from the binary inspiral of neutron stars and black holes}

One of the primary goals of the new generation of laser interferometric gravitational wave detectors is the detection of gravitational waves from the binary inspiral of compact objects, specifically neutron stars (NS) and black holes (BH's). There is a large literature written on the subject of matched filtering for detection of gravitational waves using laser interferometers (see e.g. [19-21]). The matched filtering techniques are based on Eq. (4), where $\tilde{h}(f)$ is the Fourier transform of the gravitational strain generated from the differential output of the interferometer, the $\tilde{h}_{s}(f)$ are the Fourier transforms of theoretically generated binary inspiral signal waveforms, and $S_{h}(f)$ is the measured power spectral density of the interferometer.

A significant amount of work has gone into the calculation of binary inspiral waveforms (called "templates"), the spacing of such templates to achieve near-optimal sensitivity, and the cost of generating such templates in terms of compute cycles and storage requirements [22,16,18,23,24]. Current matched filter techniques require thousands to tens of thousands of templates to cover the space of expected waveforms depending on the mass range of the binary systems considered.

The method of chirp transforms described here does away with the requirement of generating thousands of individual templates and provides a natural way to cover the space of allowed waveforms completely. To apply the chirp transform to the binary inspiral problem, we make use of the stationary phase formalism. Droz et al. [14] have shown that the stationary phase formalism can be used to provide an accurate approximation to the Fourier transform of the time-domain waveforms of inspiraling binaries as calculated in the "Newtonian" approximation. This is essentially an application of the stationary phase approximation (SPA) discussed in the Appendix to the case of gravitational waveforms. Damour et al. $[25,15]$ have shown that the binary inspiral waveforms can be accurately calculated using the SPA and an alternative formalism based on " $P$-approximants." They note that care must be taken in the treatment of the termination of the waveform at the time of the final plunge and merger.

In order to illustrate the use of the FCT in gravitational wave detection, we discuss the example of a "postNewtonian", (PN) expansion. The canonical PN stationary phase waveforms for binary inspiral up to $2 \mathrm{PN}$ order are of the form [18]

$$
\begin{aligned}
\tilde{h}_{s}(f)= & \left(\frac{5 \mu}{96 \mathrm{M}_{\odot}}\right)^{1 / 2}\left(\frac{M_{t o t}}{\pi^{2} \mathrm{M}_{\odot}}\right)^{1 / 3} f^{-7 / 6} \mathrm{~T}_{\odot}^{-1 / 6} \exp [i \Psi(f)] \\
\Psi(f)= & 2 \pi f t_{c}-2 \phi_{c}-\pi / 4+\frac{3}{128 \eta}\left[\chi^{-5 / 3}+\left(\frac{3715}{756}+\frac{55}{9} \eta\right)\right. \\
& \times \chi^{-1}-16 \pi \chi^{-2 / 3}+\left(\frac{15293365}{508032}+\frac{27145}{504} \eta\right. \\
& \left.\left.+\frac{3085}{72} \eta^{2}\right) \chi^{-1 / 3}\right],
\end{aligned}
$$

where, for simplicity, we consider only one polarization. The variables have been defined as usual $\left(\mathrm{M}_{\odot}\right.$ is the mass of the sun, $\mathrm{T}_{\odot}$ is $G \mathrm{M}_{\odot} / c^{3}$ and has a value of approximately $4.925 \times 10^{-6} \mathrm{~s}, M_{t o t}$ is the total mass of the binary system, $\eta=\mu / M_{\text {tot }}, \mu$ is the reduced mass of the binary, $t_{c}$ is the time of coalescence, $\phi_{c}$ is the phase at coalescence), and we have defined

$$
\chi=\frac{\pi M_{t o t} \mathrm{~T}_{\odot}}{\mathrm{M}_{\odot}} f .
$$

It can be seen that the stationary phase waveforms have frequency dependent amplitudes and phase functions that are expansions in powers of the frequency, $f$. In particular,

$$
\Psi(f)=\alpha+2 \pi t_{c} f+\lambda_{0} f^{-5 / 3}+\lambda_{1} f^{-1}+\lambda_{1.5} f^{-2 / 3}+\lambda_{2} f^{-1 / 3},
$$

where $\alpha$ is a phase constant and the $\lambda_{x}$ are coefficients of the frequency expansion which depend on $M_{t o t}, \mathrm{M}_{\odot}, \mathrm{T}_{\odot}$, and $\eta$.

In order to apply the FCT, we construct the discrete version of the matched filter output, Eq. (7), where $\tilde{h}\left(k_{0}\right)$ is the Fourier transform of the discretely sampled interferometer strain output, $\widetilde{h}_{s}\left(k_{0}\right)$ are the stationary phase waveforms given in Eq. (34) above, and $S_{h}\left(k_{0}\right)$ is the noise power spectral density of the interferometer.

The FCT is then used to evaluate the matched filter, with a resulting output,

$$
C_{\left\{t_{c}, \lambda_{0}, \lambda_{1}, \lambda_{1.5}, \lambda_{2}\right\}} \cdot
$$

In this expression, $2 \pi t_{c}$ is the conjugate variable of the linear $f$ term; $\lambda_{0}$ is the conjugate variable to the Newtonian 
term, $f^{-5 / 3}, \lambda_{1}$ is the conjugate variable to the $1 \mathrm{PN}$ term, $f^{-1}$, etc. Numerous considerations arise in selecting the search ranges for the conjugate parameters. As discussed by several authors $[23,18]$, spinless low-mass binaries should be reasonably well detected by a three parameter search over $\left\{t_{c}, \lambda_{0}, \lambda_{1}\right\}$. The conjugate parameters $\lambda_{0}$ and $\lambda_{1}$ fulfill a function similar to the parameters $\tau_{0}$ and $\tau_{1}$ that appear in the literature (e.g. $[23,18]$ ) which represent the Newtonian and $1 \mathrm{PN}$ contributions to the time to coalescence, respectively. Thus, in the case of spin-less low-mass binaries, $\lambda_{\{1.5,2\}}$ can be considered functions of $\lambda_{\{0,1\}}$. Owen and Sathyaprakash [18] point out that $\tau_{\{0,1.5\}}$ may be more convenient search parameters. Hence, $\lambda_{\{1,2\}}$ become functions of $\lambda_{\{0,1.5\}}$. In this case, the "heterodyne" approach [see the discussion following Eq. (31)] can be used with the dependent parameters to reduce the search space to that of spinless $2 \mathrm{PN}$ waveforms.

To search for binaries with spin, additional independent parameters will be needed and thus it will be useful to search in a range around the spin-less PN expansion (or other expansion). This can also be accomplished using the method described in the discussion following Eq. (31). The technique will be particularly useful for massive binaries for which spin interactions could be significant. An important step will be to estimate limits to the range of the conjugate variables in the FCT analysis due to spin effects. The FCT then provides a formalism for searching the complete waveform space, even if the exact waveforms are not known.

It will also be quite useful to enlarge the search region beyond the space physically accessible by astrophysical binary systems. While no binaries are expected outside the physically accessible regions, it is important to study the characteristics of noise signals in regions close to the physically accessible regions. The FCT formalism provides a straightforward way to tailor the analysis to a range of search regions. This, of course, is also possible with conventional template-based techniques.

The FCT formalism may be useful for expansions other than post-Newtonian. In particular, we are very interested to see whether the FCT formalism can be applied to the $P$-approximants discussed very recently by Damour et al. [15]. Also, as we remarked earlier, the FCT lends itself naturally to hierarchical approaches for binary inspiral detection. We note in particular the recent paper by Tanaka and Tagoshi [24] which discusses efficient hierarchical search algorithms which have several similarities to the general FCT algorithm.

In summary, the use of the FCT for detection of the chirps from gravitational waves has several attractive features. First, no explicit calculation and storage of gravitational waveforms is required for the analysis. Only the order of the $\mathrm{PN}$ expansion, the power-law exponents appearing in the expansion, and the range of the search parameters is important. Second, waveforms with perturbations on the phase evolution such as those due to spins can be detected even if the exact waveforms are not known since the FCT can be used to search completely an arbitrary region of parameter space. The only requirement is that the perturbation not involve significant terms beyond those in the expansion considered for the FCT. The FCT may therefore be very useful in the search for $\mathrm{BH}-\mathrm{BH}$ coalescence where the waveforms are not precisely known [26], or for sources to be detected by the space-based gravitational detector, the Laser Interferometer Space Antenna (LISA), where the waveforms are also only approximately known and phase perturbations are likely to be present. Finally, the FCT formalism can be used to investigate the characteristics of noise signals in the neighborhood of expected signals from binary inspiral.

\section{B. Detection of rotating neutron stars}

\section{Acceleration searches}

Pulsars are rotating neutron stars that spin with periods in the range of approximately $1 \mathrm{~ms}$ up to hundreds of seconds. Pulsars are detected primarily at radio and $x / \gamma$-ray wavelengths. In the future, rotating neutron stars may also be detectable as sources of gravitational waves. Detection of pulsars usually employs Fourier transform techniques to find the periodic pulses. However, several effects complicate the detection of pulsars and cause the pulsations to deviate from being strictly periodic. For instance, the emission from pulsars in compact binary systems is Doppler shifted causing a frequency variation on the time scale of the orbital period. Likewise, the earth's rotation and orbit can induce frequency and phase variations that are dependent on the position of the source on the sky. Rotating neutron stars can also have nonnegligible spin down effects, especially if the neutron star is young. Any of these effects can be important at both radio and $\mathrm{x} / \gamma$-ray wavelengths depending on the length of the observation. They are also likely to be important in future searches for gravitational wave emission from rotating neutron stars due to $r$-modes, or from older rotating neutron stars because of the earth's orbit and rotation.

In the past, so-called "acceleration searches" have been employed to detect pulsars with slowly varying frequency [8]. These are essentially matched filter techniques implemented either with templates, or equivalently, with "stretching' of the time or frequency variable. This requires individual matched filter operation, one for each discrete acceleration trial. The FCT analogue is that of the quadratic chirp analysis discussed in Sec. III. The FCT also provides a natural extension to searches beyond quadratic (acceleration) effects.

\section{Dispersion measure searches}

Radio radiation emitted by pulsars travels through a diffuse interstellar plasma known as the interstellar medium (ISM) before reaching detectors on Earth. The dispersive properties of the ISM cause individual radio pulses to broaden in time. This dispersion broadening will reduce the chances of detecting a given pulsar signal. The magnitude of the dispersion effect is measured by a quantity called the dispersion measure (DM). If the DM is known, the dispersion effect can be removed from the pulsar signal using standard digital signal processing techniques. When searching for new pulsars, the DM is rarely known and systematic searches must be performed both in DM and in the pulsar period. 
The effect of ISM dispersion may be removed from the received signal by applying the following transformation $[27,7]$ :

$$
S(D M, t)=\int_{-\infty}^{\infty} \hat{S}_{r}(f) e^{i 2 \pi f t} e^{i 2 \pi D M \phi(f)} d f,
$$

where $\hat{S}_{r}(f)$ is the Fourier transform of the received signal and $S(D M, t)$ is known as the dedispersed signal. When searching for new pulsars, one must generate several time series corresponding to different values of the DM. The above equation shows that $S(D M, t)$ is simply a chirp transform of $\hat{S}_{r}(f)$. The FCT provides an efficient way to generate $S(D M, t)$ for several values of DM. Each of these time series can then be searched for periodic signals.

When searching for pulsar signals, one typically "detects" the total power in the signal by calculating $P(D M, t)=\|S(D M, t)\|^{2}$ and then averages over a small window of time. Each time series is then searched separately using various pulsar detection techniques. The structure of the FCT points to the possibility of a slightly different technique. Rather than searching each time series separately, one first calculates $P(t)=\Sigma_{D M} P(D M, t)$ and then searches this time series for possible pulsar signals (see Sec. III B). Using the property that the sum of the squares is conserved under a Fourier transform, the second set of FFT's in the FCT does not need to be performed in order to calculate $P(t)$. Thus, a highly efficient intermediary chirp transform can be used instead of the complete FCT.

\section{SUMMARY}

We have described an algorithm for the detection of signals with variable frequency. Standard detection algorithms use matched filtering techniques which require both the computation of a large set of task specific filter functions and a prescription for densely covering the set of possible signal waveforms. The fast chirp transform proposed in this paper automatically precludes the need to generate specific filter functions since standard FFT's can be used in the implementation and the FCT immediately provides the prescription for densely covering the waveform parameter space.

The FCT for a two parameter chirp was defined and then generalized to $N$ parameters with arbitrary phase functions. A straightforward implementation of the FCT was discussed and it was shown to be comparable in efficiency with the brute-force matched filtering approach. Several approaches to achieving even better computational efficiency were also discussed.

The efficient detection of variable frequency signals has a large number of practical applications. Of considerable interest to the authors is the detection of gravitational waves from NS and BH binary systems and the detection of radio waves from pulsars. Another obvious area of application is radarsonar signal processing where target or transmitter motion can cause Doppler frequency shifts in the received signal. Other potential areas of application include communications and image processing. A more detailed description of the
FCT and its application to the above problems will be the subjects of future work.

\section{ACKNOWLEDGMENTS}

We acknowledge informative and pivotal discussions with Ben Owen and B. Sathyaprakash. We also acknowledge helpful and stimulating discussions with Alessandra Buonanno, Tibault Damour, Scott Hughes and Albert Lazzarini. This work was supported in part by the LIGO Laboratory under cooperative agreement NSF-PHY-9210038 and by grant NSF-PHY-9970877. This paper is LIGO document LIGO P000003-00-R.

\section{APPENDIX: THE STATIONARY PHASE APPROXIMATION AND MATCHED FILTERING}

\section{Frequency-domain matched filtering}

We begin by showing how the Fourier transform of waveforms such as those of Eq. (1) can be approximated in a way that allows them to be expressed naturally in frequencydomain matched filtering, Eq. (4). The stationary phase approximation (see e.g. [28]; see also Ref. [19] and Refs. $[29,15]$ for a description in the context of gravitational wave detection), provides a prescription for approximating the Fourier transform of a function of the form $h_{s}(t)$ $=A(t) \cos (\phi(t))$ [where $A(t)$ and $\phi(t)$ are real and $\phi^{\prime}(t)$ is positive]:

$$
\widetilde{h}_{s}(f)=\frac{1}{2}\left[\int_{-\infty}^{\infty} d t A(t) e^{i f \psi_{+}(t)}+\int_{-\infty}^{\infty} d t A(t) e^{-i f \psi_{-}(t)}\right]
$$

with $\psi_{ \pm}(t)=\phi(t) / f \pm 2 \pi t$. If $t_{f}$ exists such that $\psi_{+}^{\prime}\left(t_{f}\right)=0$ or $\psi_{-}^{\prime}\left(t_{f}\right)=0$, then $t_{f}$ is called a "stationary point." Considering positive $f$ and positive $\phi^{\prime}(t)$, only the second integral in Eq. (A1) contains a stationary point. Hence, to leading order, we can write [28]:

$$
\tilde{h}_{s}(f) \sim \frac{1}{2} \int_{-\infty}^{\infty} d t A(t) e^{i[2 \pi f t-\phi(t)]}
$$

for $f>0$. Note that we compute $\widetilde{h}_{s}(-f)$ using the Fourier transform property of real functions: $\widetilde{h}_{s}(-f)=\widetilde{h}_{s}^{*}(f)$.

If all derivatives of $\psi_{-}(t)$ up to order $p$ are zero at $t_{f}$, then the Fourier transform of $h_{s}$ may be approximated by

$$
\widetilde{h}_{s}(f) \sim \mathcal{A}(f) \exp [i \Psi(f)],
$$

with components given by somewhat complicated but straightforward expressions:

$$
\mathcal{A}(f)=A\left(t_{f}\right)\left[\frac{p !}{f\left|\psi^{(p)}\left(t_{f}\right)\right|}\right]^{1 / p} \frac{\Gamma(1 / p)}{p},
$$

and

$$
\Psi(f)=2 \pi f t_{f}-\phi\left(t_{f}\right) \pm \pi / 2 p,
$$

where the sign of $\pi / 2 p$ is positive or negative depending on whether $\psi_{-}^{(p)}\left(t_{f}\right)$ is positive or negative, respectively. In particular, for $p=2$, the following approximation holds: 


$$
\tilde{h}_{s}(f)=\sqrt{\frac{\pi}{2}} A\left(t_{f}\right)\left|\phi^{\prime \prime}\left(t_{f}\right)\right|^{-1 / 2} e^{i\left[2 \pi f t_{f}-\phi\left(t_{f}\right) \pm \pi / 4\right]} .
$$

The stationary phase approximation is accurate as long as the amplitude of $h_{s}$ does not vary too quickly compared to the time derivative of the phase, $\phi^{\prime}(t)$, and the effect of the higher derivatives of $\phi(t)$ on the phase evolution are small compared to the effect of $\phi^{\prime}(t)$.

Using the form given in Eq. (A3), we can now rewrite Eq. (4). Gathering all the amplitude terms together,

$$
\tilde{\mathcal{H}}(f)=\tilde{h}(f) \frac{\mathcal{A}^{*}(f)}{S_{h}(f)}
$$

we can express the matched filter output as

$$
\mathcal{S}\left(t_{0}\right)=4 \operatorname{Re}\left[\int_{0}^{\infty} d f \tilde{\mathcal{H}}(f) e^{-i \Phi(f)}\right],
$$

where

$$
\Phi(f)=\Psi(f)-2 \pi f t_{0} .
$$

Hence, the matched filtering operation in the frequency domain is expressed as an integral transform, specifically a socalled generalized Fourier integral. In analogy with the discrete Fourier transform (DFT), we can write this in discrete form as

$$
\mathcal{S}=\frac{4}{N_{0}} \operatorname{Re}\left[\sum_{k_{0}=0}^{N_{0}-1} \tilde{\mathcal{H}}_{k_{0}} e^{-i \Phi_{k_{0}}}\right]
$$

The summation can be computed as a FCT.

\section{Time-domain matched filtering}

We note that for signal waveforms of the form, $h_{s}(t)$ $=A(t) \cos (\phi(t))$, the expression [Eq. (2)] for time-domain matched filtering yields directly

$$
\mathcal{S}=\operatorname{Re}\left[\int_{0}^{T} d t h(t) A(t) e^{i \phi(t)}\right] .
$$

Such signals are of considerable interest and include periodic signals with frequency drift. The integral transform in Eq. (A11) can be represented in discrete form in the usual way as

$$
\mathcal{S}=\operatorname{Re}\left[\sum_{j_{0}=0}^{N_{0}-1} \mathcal{G}_{j_{0}} e^{i \phi\left(j_{0}\right)}\right],
$$

where

$$
\mathcal{G}_{j_{0}}=h\left(j_{0}\right) A\left(j_{0}\right)
$$

and where $h\left(j_{0}\right), A\left(j_{0}\right)$, and $\phi\left(j_{0}\right)$ are the discretely sampled values of the continuous functions.
[1] J. S. Lim and A. V. Oppenheim, Advanced Signal Processing (Prentice Hall, New Jersey, 1988).

[2] R. Carmona, W. Hwang, and B. Torresani, Practical TimeFrequency Analysis (Academic, San Diego, 1998).

[3] C. Helstrom, Statistical Theory of Signal Detection (Pergamon, Oxford, 1968).

[4] S. M. Kay, Statistical Signal Processing (Prentice Hall PTR, New Jersey, 1993).

[5] E. T. Jaynes, in Maximum-Entropy and Bayesian Spectral Analysis and Estimation, edited by C. Smith and G. J. Erickson (Reidel, Dordrecht, 1987), pp. 1-37.

[6] H. L. Van Trees, Detection, Estimation, and Modulation Theory (Wiley, New York, 1968).

[7] F. A. Jenet, W. R. Cook, T. A. Prince, and S. C. Unwin, Publ. Astron. Soc. Pac. 109, 707 (1997).

[8] S. B. Anderson, Ph.D. thesis, California Institute of Technology, 1993.

[9] S. Smith, Phys. Rev. D 36, 2901 (1987).

[10] A. V. Oppenheim, R. W. Schafer, and J. R. Buck, DiscreteTime Signal Processing (Prentice Hall, New Jersey, 1999).

[11] B. F. Schutz, in Proceedings of the Second Workshop on Gravitational Wave Data Analysis, edited by M. Davier and P. Hello (Editions Frontieres, Paris, 1998), pp. 133-143, gr-qc/9802020.

[12] P. Williams and B. Schutz, in "Proceedings of the 3rd Edoardo Amaldi Conference, 1999,"' gr-qc/9912029.

[13] D. Bailey and P. Swarztrauber, SIAM Rev. 33, 389 (1991).

[14] S. Droz, D. Knapp, E. Poisson, and B. Owen, Phys. Rev. D 59, 124016 (1999).
[15] T. Damour, B. R. Iyer, and B. S. Sathyaprakash, Phys. Rev. D 62, 084036 (2000).

[16] B. Owen, Phys. Rev. D 53, 6749 (1996).

[17] S. Mohanty and S. Dhurandhar, Phys. Rev. D 54, 7108 (1996).

[18] B. Owen and B. Sathyaprakash, Phys. Rev. D 60, 022002 (1999).

[19] K. Thorne, in 300 Years of Gravitation, edited by W. Israel and S. W. Hawking (Cambridge University Press, Cambridge, England, 1987), pp. 330-458.

[20] B. Schutz, in The Detection of Gravitational Waves, edited by D. Blair (Cambridge University Press, Cambridge, England, 1991), pp. 406-452.

[21] B. Allen, GRASP: A Data Analysis Package for Gravitational Wave Detection, http:/www.lsc-group.phys.uwm.edu/ $\sim$ ballen/grasp-distribution, v1.9.7 ed. (1999).

[22] T. A. Apostolatos, Phys. Rev. D 52, 605 (1995).

[23] S. Mohanty, Phys. Rev. D 57, 630 (1998).

[24] T. Tanaka and H. Tagoshi gr-qc/0001090, 2000.

[25] T. Damour, B. Iyer, and B. Sathyaprakash, Phys. Rev. D 57, 885 (1998).

[26] P. Brady, J. Creighton, and K. Thorne, Phys. Rev. D 58, 061501 (1998).

[27] T. H. Hankins and B. J. Rickett, in Methods in Computational Physics. Radio Astronomy (Academic, New York, 1975), Vol. 14, pp. 55-129.

[28] C. Bender and S. Orszag, Advanced Mathematical Methods for Scientists and Engineers (McGraw-Hill, New York, 1978).

[29] L. Finn and D. Chernoff, Phys. Rev. D 47, 2198 (1993). 